\title{
Dynamic characterization of multi-component sensors for force and moment
}

\author{
Jan Nitsche ${ }^{1,2}$, Rolf Kumme ${ }^{1}$, and Rainer Tutsch ${ }^{2}$ \\ ${ }^{1}$ Physikalisch-Technische Bundesanstalt, Bundesallee 100, 38116 Braunschweig, Germany \\ ${ }^{2}$ Technische Universität Braunschweig, Institut für Produktionsmesstechnik, Schleinitzstr. 20, \\ 38106 Braunschweig, Germany \\ Correspondence: Jan Nitsche (jan.nitsche@ptb.de)
}

Received: 21 August 2018 - Revised: 10 October 2018 - Accepted: 15 October 2018 - Published: 7 November 2018

\begin{abstract}
An improved set-up for the characterization of multi-component sensors for force and moment is presented. It aims at calibrating such sensors under continuous sinusoidal excitation. Special focus is put on the design of load masses and adapting elements to activate uniaxial force and moment components where possible. To identify the motion and acceleration of the load mass with 6 degrees of freedom, a photogrammetric measurement system is implemented in the existing set-up. Using the set-up described, different experiments are performed to analyse a commercial multi-component sensor and perform a parameter identification for its force components.
\end{abstract}

\section{Introduction}

The calibration and characterization of sensors for force and moment are typically performed in a static manner (ISO 376, 2011; DIN 51309, 2005). The dynamic behaviour of these sensors may differ significantly from the static behaviour. Especially the sensitivity coefficient, which is necessary to transform the sensor output signal into a force or moment signal, is subject to changes with higher frequencies of the dynamic excitation (Schlegel et al., 2012; Vlajic and Chijioke, 2016). Therefore, if such sensors are to be used in a dynamic environment, their dynamic behaviour needs to be analysed (Bartoli et al., 2012). For force and acceleration sensors, typical characterization methods are sinusoidal excitation (Schlegel et al., 2012) and shock excitation (Bruns et al., 2002). Torque sensors are currently evaluated under sinusoidal excitation (Klaus, 2016).

The aforementioned calibration approaches are limited to uniaxial sensors or calibrations of only one axis of multiaxial or multi-component sensors (MCSs). Such MCSs have become more popular in the last few years, which has resulted in the need for new calibration procedures (Kim, 2000; Baumgarten et al., 2016; Nitsche et al., 2017a). Challenges in the calibration of MCSs are, among other things, the generation of forces and moments with defined directions, the identification of the force and moment vector in a reference system (Röske et al., 2001) and the alignment of sensor and reference coordinate systems (Nitsche et al., 2017b).

For the dynamic calibration of MCSs, very few approaches exist. Park et al. (2008) deployed an electrodynamic shaker set-up with a vertical excitation direction at the PhysikalischTechnische Bundesanstalt (PTB) using different adapting elements to activate sinusoidal excitations in different directions. Schleichert et al. (2016) designed a fixed sensor frame with different adapting directions for an electrodynamic plunger actuator. In both set-ups, the reference force can only be calculated in the direction of excitation. Additional components like rocking modes cannot be detected.

In the following sections, an improved set-up for the dynamic analysis of MCSs is described. The force generation is based on the periodic acceleration of a load mass connected to an MCS. The theory behind sinusoidal calibration is explained using the example of uniaxial force calibration in Sect. 2. The improved set-up includes a specially designed load mass and a three-dimensional acceleration reference based on photogrammetry. Section 3 summarizes the new set-up. A dynamic model of an MCS is described in Sect. 4. Finally, the parameters of the model are identified from experimental data as described in Sect. 5. 


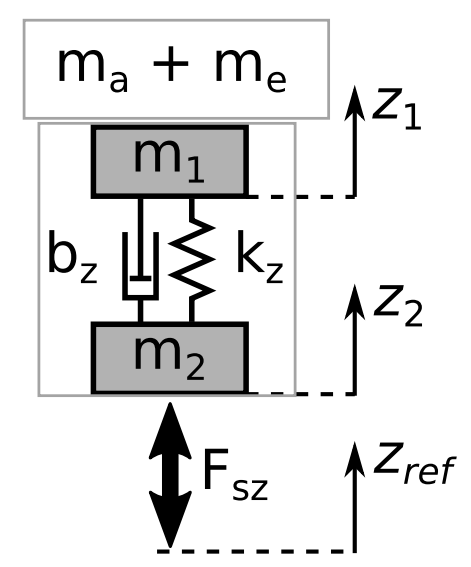

Figure 1. Dual-mass oscillator as a model for uniaxial force sensors.

\section{Sinusoidal calibration of force and moment sensors}

The aim of the dynamic calibration of force and moment sensors is the identification of the parameters needed to describe the dynamic behaviour and the sensitivity coefficient as a function of the excitation frequency (Schlegel et al., 2012). The typical model used for the description of such a sensor is a mass-spring-damper system. The parameters needed to describe this model are the spring constant $k_{z}$, the damping parameter $b_{z}$ and the mass $m$ (Fig. 1). The mass $m$ is composed of the external load mass $m_{\mathrm{e}}$, the mass of adapting elements $m_{\mathrm{a}}$ and the internal moving mass of the sensor $m_{1}$.

From the given parameters, the dynamic sensitivity $S$ can be calculated according to Schlegel et al. (2012):

$S=\frac{c}{\sqrt{k_{z}^{2}+\left(b_{z} \omega\right)^{2}}} \approx \frac{c}{k_{z}}\left(1-\frac{1}{2}\left(\frac{b_{z}}{k_{z}}\right)^{2} \omega^{2}\right)$,

with $c$ as a factor for unit conversion with the unit $\mathrm{Um}^{-1}$ (U as a sensor signal unit, e.g. $\mathrm{mVV}^{-1}$ for strain gauge sensors). In addition to the sensitivity $S$, the phase shift $\varphi_{\mathrm{s}}$ between the excitation and the sensor output can be calculated as follows:

$\varphi_{\mathrm{s}} \approx-\frac{b_{z}}{k_{z}} \omega$.

Both Eqs. (1) and (2) are approximations for a stiff coupling of the top mass to the sensor, which allows the influence of an additional spring-damper system describing the coupling to be neglected.

As an alternative to identifying the model parameters $k$ and $b$, the dynamic sensitivity can be calculated as the ratio of the sensor signal output $U$ and the acting dynamic force $F$ (Park et al., 2008):

$S=\frac{U}{F}$.
In this case, only the moving mass $m$ and its acceleration $a$ are needed to calculate the acting force:

$F=m \cdot a$.

\section{Measurement set-up}

The measurement set-up for the periodic excitation of MCSs used in this work is based on the shaker set-up located in the dynamic force calibration laboratory at the PTB (Schlegel et al., 2012). It consists of an LDS $10 \mathrm{kN}$ electrodynamic shaker for frequencies up to $2 \mathrm{kHz}$ and a laser scanning vibrometer as an acceleration reference. Different accelerometers can be used as additional acceleration references.

The electrodynamic shaker is only capable of generating accelerations in one direction. To activate force and moment components in all six directions, different adapting elements are needed. The design of such elements is described in Sect. 3.1.

The scanning vibrometer measures accelerations in the direction of the excitation of the shaker system. Accelerations perpendicular to the excitation direction, as well as twisting motions of the load mass, cannot be detected. To rectify this, a photogrammetric measurement system is used to detect accelerations that are not visible to the scanning vibrometer. Details of the photogrammetric system, synchronization and data analysis are described in Sect. 3.2.

\subsection{Load masses and adapting elements}

In past works (Park et al., 2002a, b, 2008), different MCSs were investigated on the previously described shaker in a similar set-up. This set-up consisted of a cylindrical load mass in combination with an air-bearing guide for axial force. Transverse force components were generated using $90^{\circ}$ angular adapters and a cubic load mass. Bending moments and torque were generated using a lever arm in combination with the aforementioned cubic load mass.

Due to its design, this set-up was only capable of generating uniaxial force components in the axial force direction $F_{z}$. Transverse force components $F_{x}$ and $F_{y}$ were superimposed by moment components $M_{y}$ and $M_{x}$ as a result of the distance between the sensor coordinate system and the centre of gravity of the external load mass. Torque components $M_{z}$ were superimposed by bending moments $M_{x}$ or $M_{y}$ and transverse forces $F_{y}$ or $F_{x}$.

To overcome these disadvantages, a special load mass was designed in order to move the centre of gravity of the load mass to the origin of the coordinate system of the sensor under test (Nitsche et al., 2018a). Using this load mass, rocking modes for the axial force component $F_{z}$ are reduced and transverse force components $F_{x}$ and $F_{y}$ can be generated without superimposed moment components. The mass of the load mass is $m_{\mathrm{L}}=(6.9605 \pm 0.0010) \mathrm{kg}$. The angular adapter for activating transverse forces is designed in order to move 


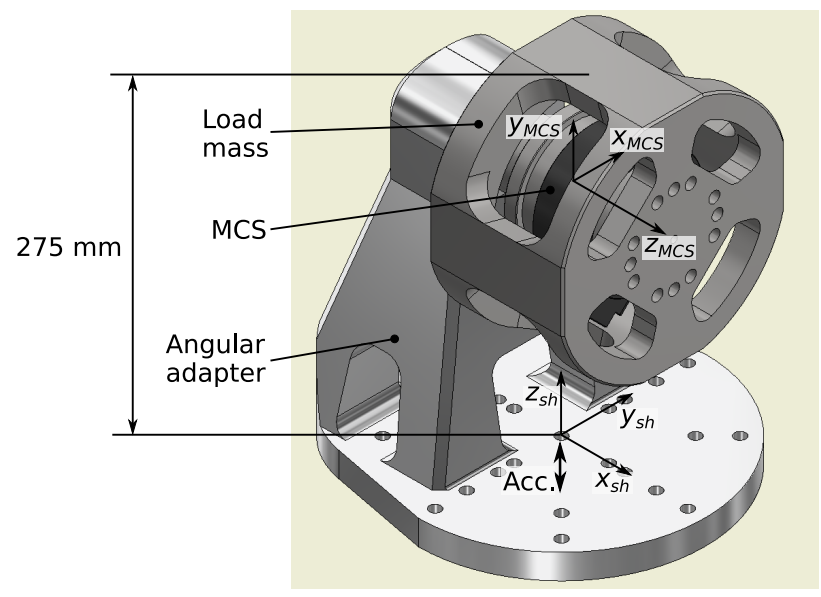

Figure 2. Load mass and adapting element for transverse force components.

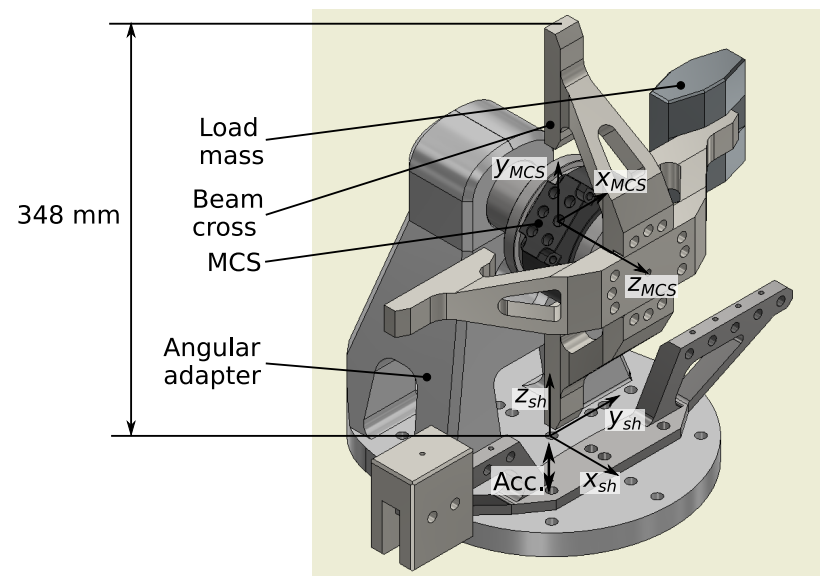

Figure 3. Beam cross for generation of moment components.

the centre of gravity of the whole set-up in the axis of the shaker table to avoid moment loads on the shaker bearings. The set-up including load mass, sensor and the adapting element for transverse force components is shown in Fig. 2.

For the generation of moment components, a beam cross is used. The cross is designed in a way to move the centre of gravity of the moment generating mass components to the $x-y$ plane of the sensor coordinate system. This results in a superposition of bending moments $M_{x}$ or $M_{y}$ with only one axial force $F_{z}$ and the superposition of torque $M_{z}$ with only one transverse force $F_{x}$ or $F_{y}$. The distance between the load mass and the sensor can be changed to generate different amplitudes of the moment component. To reduce moment loads on the shaker bearing, a counter mass is attached to the base of the angular adapter. The beam cross set-up is shown in Fig. 3.

\subsection{Three-dimensional acceleration reference}

To evaluate whether signal outputs of inactive force or moment components of the sensor are a result of signal crosstalk from the active component or of bending or twisting moments, a complete three-dimensional movement of the set-up needs to be identified. The scanning vibrometer is capable of measuring the acceleration in the direction of the shaker axis at different points of the set-up. From the acceleration distribution, rocking modes around the $x$ and $y$ axes can be identified. Three-dimensional accelerometers can be added to specific points in the system to obtain three-dimensional acceleration information at that point. However, the mass of the accelerometer and its cable has an influence on the dynamic behaviour of the whole set-up.

To extend the acceleration measurement provided by the laser vibrometer and the accelerometers, a photogrammetric measurement set-up is installed. It consists of two stereo camera systems: one camera system observing the load mass on top of the sensor, the other one directed at the surface of the shaker. All four cameras are triggered simultaneously using a signal generator. One master camera is used to trigger two LED strobe lights to illuminate the observed area. This trigger signal is recorded by the analogue to digital converter (ADC) in the junction box of the data acquisition PC of the scanning vibrometer for synchronization purposes. The trigger signal also serves as the time stamp of the image acquisition. The six force and moment signals of the sensor under test are amplified by a 6-channel bridge amplifier (Dewetron, 2012), digitized by a simultaneously sampling 16-channel ADC card (National Instruments, 2012) and saved on a separate data acquisition PC. The camera trigger signal is also recorded by this $\mathrm{PC}$ for synchronization. A block diagram of the extended shaker set-up is shown in Fig. 4. A photograph of the sensor with the attached load mass, the shaker and the stereo camera systems is shown in Fig. 5.

Random greyscale patterns are attached to the surfaces observed by the cameras. The images of these patterns are analysed using digital image correlation (DIC) algorithms (Pan et al., 2009). In DIC, the camera images are split into small areas and the correlation between the areas in different camera images is calculated. In this way, one area of the observed surface can be identified in the images of both cameras and at different times. Each stereo set-up is able to calculate the position of multiple areas on the observed surface in space. Those three-dimensional points can be used to calculate a three-dimensional rigid body movement as well as a deformation of the load mass in the observed area.

The rotation and translation of the rigid body transformation are calculated using the singular value decomposition (SVD) method presented by Arun et al. (1987) (Eggert et al., 1997). From the resulting rotation matrix, the rotation angles are calculated in a fixed axis system. This assumption can be used as the rotations around any axis are expected to be very small and therefore do not influence each other. 


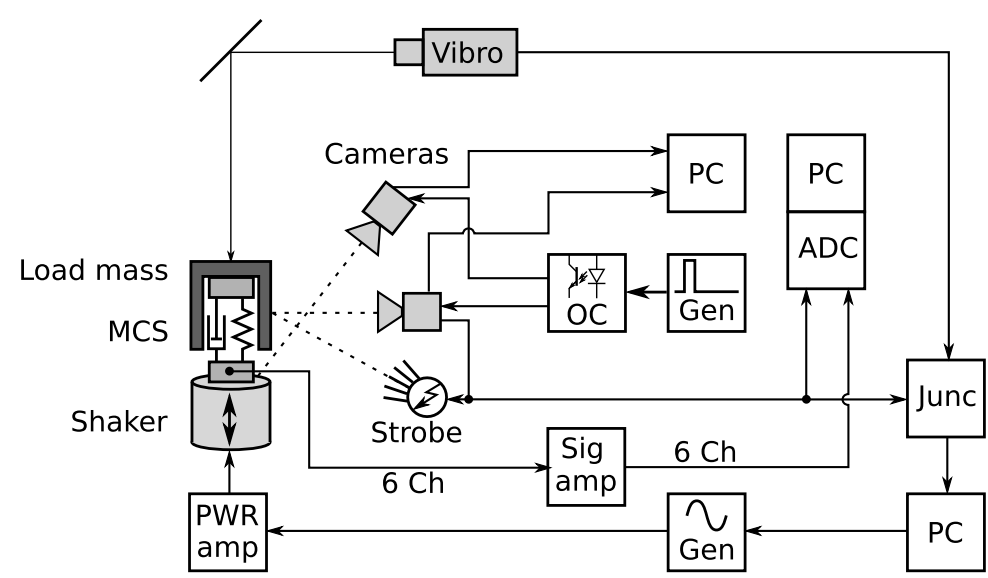

Figure 4. Block diagram of the photogrammetric set-up in the dynamic shaker system.

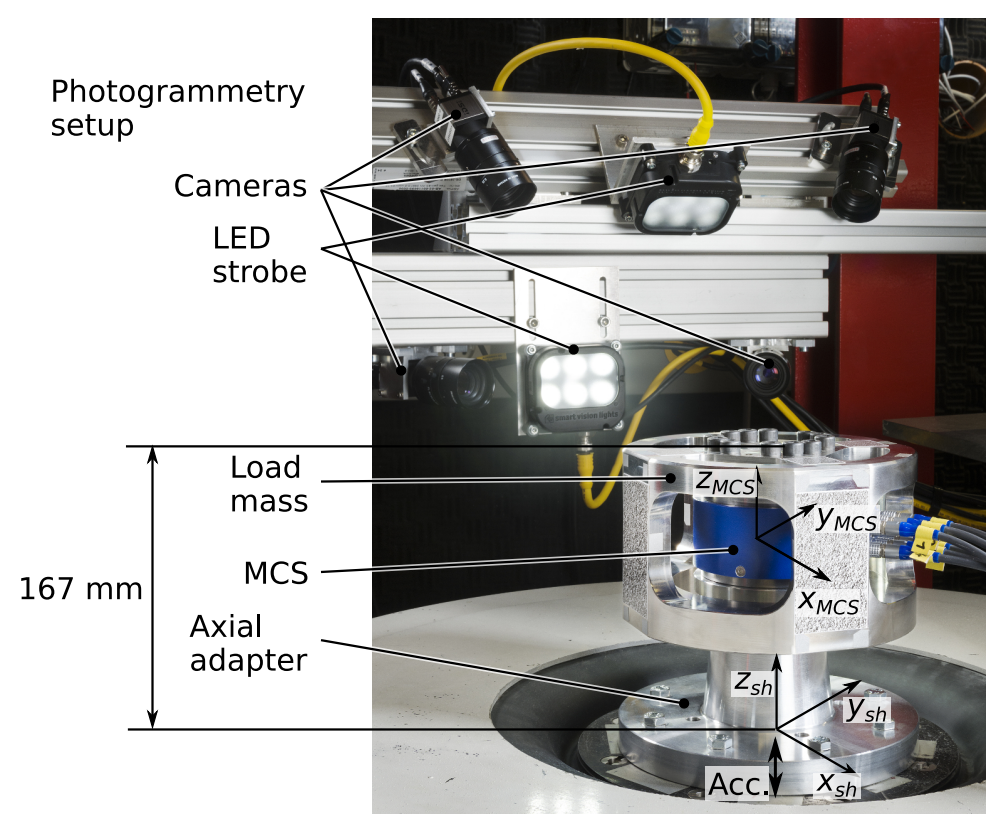

Figure 5. Photogrammetric set-up in the shaker environment.

The cameras used for DIC are industrial CMOS cameras with frame rates of up to 166 images per second for 8-bit greyscale images. At 12-bit colour depth, the frame rate is reduced to a maximum of 82 images per second. This frame rate is too low to satisfy the Nyquist-Shannon sampling theorem for frequencies higher than $40 \mathrm{~Hz}$. However, using the additional knowledge of the excitation frequency of the shaker and the precise adjustment of the sampling rate of the cameras, both frequencies can be adjusted to achieve a beat frequency.

The known frequency distribution resulting from the vibrometer measurement is used to define a fitting function for the displacement of the load mass $S(t)$ :

$S(t)=\sum_{i}\left(\hat{s}_{i} \cdot \sin \left(2 \cdot \pi \cdot f_{\mathrm{i}} \cdot t+\varphi_{i}\right)\right)+o$,

with $f_{\mathrm{i}}$ as the identified frequencies of the frequency distribution, $\hat{s}_{i}$ the amplitude, $\varphi_{i}$ the phase shift of each frequency and $o$ the offset of the mean. The parameters $\hat{s}, \varphi$ and $o$ can be estimated by a least squares fitting algorithm. The resulting function $S(t)$ represents the axial or angular displacement of the load mass over time.

\subsection{Experimental analysis of the set-up}

An experimental evaluation of the set-up described is performed using the axial force components. The excitation fre- 

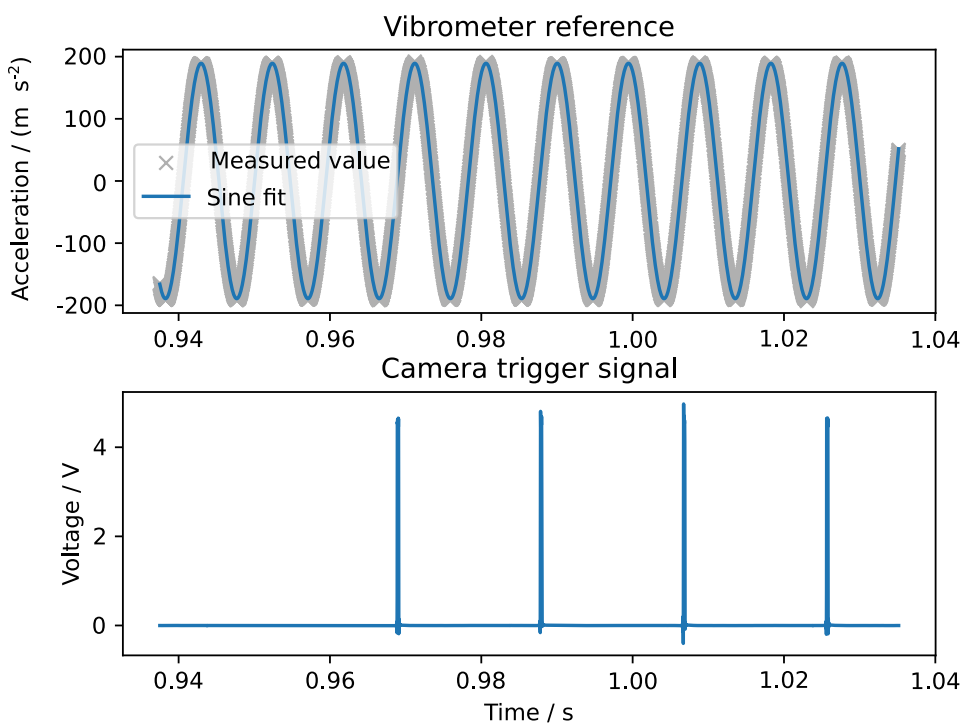

Figure 6. Vibrometer and camera trigger signals.

Table 1. Fitted sine parameters.

\begin{tabular}{lll|ll}
\hline & \multicolumn{2}{c|}{ Vibrometer } & \multicolumn{2}{c}{ Photogrammetry } \\
\hline Parameter & Freq. 1 & Freq. 2 & Freq. 1 & Freq. 2 \\
\hline Frequency & $106.2503 \mathrm{~Hz}$ & $425.0000 \mathrm{~Hz}$ & $106.2495 \mathrm{~Hz}$ & $424.9472 \mathrm{~Hz}$ \\
Amplitude & $189.3217 \mathrm{~m} \mathrm{~s}^{-2}$ & $0.9596 \mathrm{~m} \mathrm{~s}^{-2}$ & $0.4277 \mathrm{~mm}$ & $-0.0040 \mathrm{~mm}$ \\
Phase & $0.369 \mathrm{rad}$ & $5.573 \mathrm{rad}$ & $0.208 \mathrm{rad}$ & $0.820 \mathrm{rad}$ \\
Mean & $0 \mathrm{~m} \mathrm{~s}^{-2}$ & & $-0.0425 \mathrm{~mm}$ & \\
\hline
\end{tabular}

quency is set to $f_{\mathrm{ex}}=106.25 \mathrm{~Hz}$. The amplitude of the acceleration signal is $0.5 \mathrm{~V}$. The laser vibrometer sampling frequency is $51.2 \mathrm{kHz}$ and the cameras are triggered with $52.874 \mathrm{~Hz}$. Figure 6 shows the acceleration signal recorded from the laser vibrometer, a fitted sine function and the camera trigger signal as a synchronization reference. The parameters of the sine fitting are listed in Table 1 . The camera trigger is started manually after starting the vibrometer recording. The trigger signal at $0.97 \mathrm{~s}$ represents the first image acquired by the cameras.

The difference between the camera frequency and the excitation frequency results in a beat of $0.502 \mathrm{~Hz}$. The first camera image is taken as a reference image against which all displacements are calculated. Each camera acquires 211 images, which results in 210 displacement measurements within $3.97 \mathrm{~s}$.

For each displacement measurement, the rigid body transformation is calculated as described in Sect. 3.2. As a result, three axial translations and three rotation angles along the coordinate system of the camera set-up are obtained. For each displacement measurement, the mean of the exposure time, which is identified from the strobe trigger signal, is used as the time of acquisition. The data sets of acquisition time and axial and angular displacement are used to fit a sine function according to the excitation frequency of the shaker.

Sine fitting is performed using a nonlinear least squares fitting function, curve fit, of the Python scipy optimize package. Starting values for the fitting frequencies are taken from the frequency spectrum of the vibrometer reference. Figure 7 shows the frequencies of the fast Fourier transform (FFT) up to $1000 \mathrm{~Hz}$.

The main contributions can be seen at the excitation frequency $f_{\mathrm{ex}}=106.25 \mathrm{~Hz}$ and its multiples $425,212.5$ and $318.75 \mathrm{~Hz}$. The amplitude at the second frequency $(425 \mathrm{~Hz})$ reaches a value of $0.5 \%$ of the amplitude of the excitation frequency.

Sine fitting of only the excitation frequency $f_{\text {ex }}=$ $106.25 \mathrm{~Hz}$ results in a standard deviation of the difference between the fitting function and the measured displacements of $0.0036 \mathrm{~mm}$. Using a fitting function of the two main frequencies 106.25 and $425 \mathrm{~Hz}$ reduces the standard deviation of the residuum to $0.0023 \mathrm{~mm}$. Additional fitting frequencies show no further improvement of the results. The displacement in $z$, measured with the photogrammetry set-up and the fitted sine function, is shown in Fig. 8. Figure 9 shows the ax- 


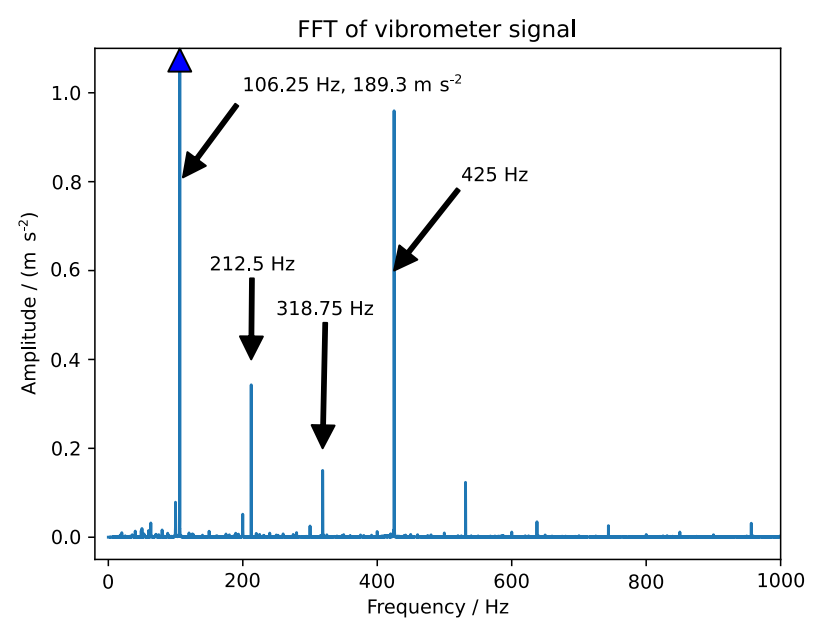

Figure 7. Frequency spectrum of the vibrometer signal. The $y$ axis is scaled to make frequencies different than the excitation frequency visible. The amplitude of $f_{\mathrm{ex}}=106.25 \mathrm{~Hz}$ is $189.3 \mathrm{~m} \mathrm{~s}^{-2}$.

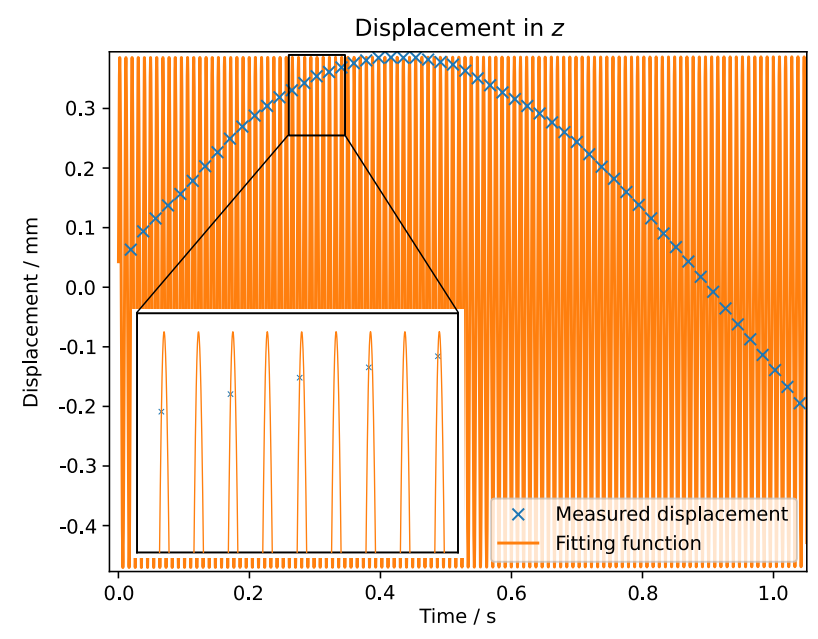

Figure 8. Displacement in $z$ from DIC and the fitted sine function.

ial and angular displacement along the $x$ axis. The resulting parameters from the sine fitting are listed in Table 1.

To calculate the resulting force and moment components on the sensor, the axial and angular acceleration of the load mass is needed. Both values can be calculated from the fitting function of the displacement as the second derivative with respect to time. For a sine function, the second derivative can easily be calculated by multiplying the function by the negative square of the angular frequency $\omega_{i}=2 \cdot \pi \cdot f_{\mathrm{i}}$. The acceleration of the fitting function of Eq. (5) results in

$\ddot{S}(t)=\sum_{i}\left(-\hat{s}_{i} \cdot \omega_{i}^{2} \cdot \sin \left(\omega_{i} \cdot t+\varphi_{i}\right)\right)$.

From the values of the fitting function listed in Table 1, the amplitude of the acceleration $\hat{a}_{i}=-\omega^{2} \cdot \hat{s}_{i}$ from the photogrammetric measurement is calculated to be $\hat{a}_{1}=$

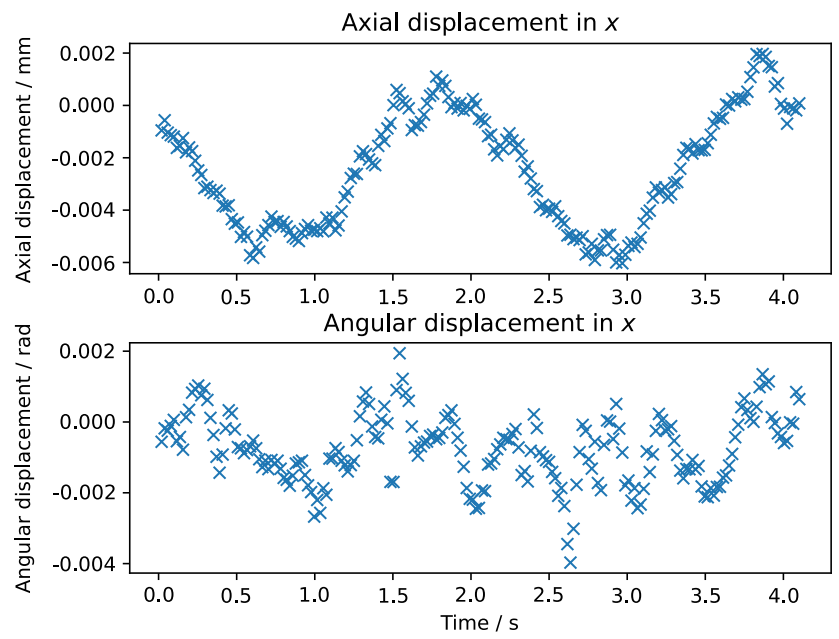

Figure 9. Axial and angular displacement in $x$.

$-190.6131 \mathrm{~m} \mathrm{~s}^{-2}$ and $\hat{a}_{2}=28.5161 \mathrm{~m} \mathrm{~s}^{-2}$. While the acceleration at the excitation frequency shows a small deviation of $0.68 \%$ to the reference value of the vibrometer, the acceleration at $425 \mathrm{~Hz}$ differs significantly. According to the reference acceleration, the amplitude of the second frequency has a minor influence on the overall acceleration and will be neglected in the subsequent analysis. It must however be included in the pending uncertainty budget.

\section{Dynamic model of multi-component sensors}

For uniaxial force sensors, a dual-mass oscillator as shown in Fig. 1 can be used as an acceptable description of the dynamic behaviour (Kumme, 1996; Schlegel et al., 2012). MCSs show a more complex structure which results in a more complex physical model. To reduce the complexity of the model, the observed six-component MCS is split into two independent models, one for the three force components, one for the moment components.

\subsection{Dynamic MC force model}

The dynamic model for a three-component force sensor is based on a superposition of three orthogonally aligned spring-damper systems connected to two masses $m_{1}$ and $m_{2}$. A simplified illustration of a two-dimensional case of this model is shown in Fig. 10. Mutual influences between the spring-damper systems are neglected. To describe this model, eight different parameters are needed: three spring constants $k_{x}, k_{y}, k_{z}$, three damping parameters $b_{x}, b_{y}, b_{z}$ and the two masses $m_{1}$ and $m_{2}$. The masses are assumed to be equal for excitation in the $x, y$ and $z$ directions. 


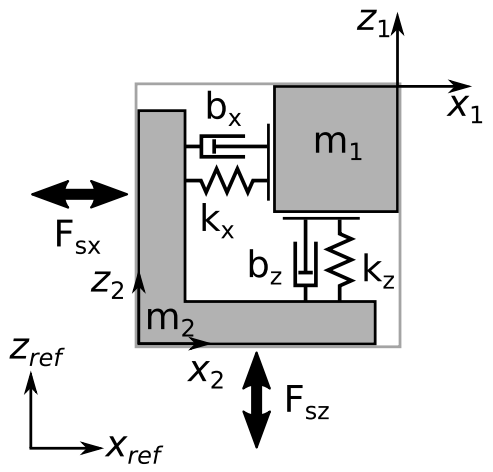

Figure 10. Two-dimensional representation of the dual-mass oscillator for multi-component force sensors.

\subsection{Dynamic MC moment model}

A dynamic model for a three-component moment sensor is more complex than the model for a three-component force sensor. Basically, the model from Fig. 10 can be adapted by changing the springs and dampers of the model from linear to rotational ones. The exchange of the masses is more difficult, as the mass moment of inertia is needed for dynamic moment measurements. This parameter depends on a rotational axis and therefore it cannot be assumed to be equal for the different excitation directions.

\section{Parameter identification}

\subsection{Determination of the internal mass}

To determine the internal mass $m_{1}$ of the sensor, the sensor is mounted onto an indexing head with a horizontal rotation axis. The $z$ axis of the sensor is aligned with the rotation axis of the indexing head. Sensor readings are recorded without an attached load mass. The sensor is rotated in steps of $22.5^{\circ}$. The sensor readings are transformed into force and moment values using the calibration matrix provided by the sensor manufacturer. A sine function is fitted to the force values for $F_{x}$ and $F_{y}$. The amplitude of the sine function represents the maximum force on each channel resulting from the internal mass of the sensor. The mass itself is calculated from the amplitude, divided by the local acceleration of gravity $g_{10 c}$ :

$m_{1}=\frac{F_{\max }}{g_{\text {loc }}}$.

Force readings and the sine fitting for $F_{x}$ and $F_{y}$ are shown in Fig. 11. The resulting amplitude of the sine function is $7.558 \mathrm{~N}$ for $F_{x}$ and $7.532 \mathrm{~N}$ for $F_{y}$, resulting in internal masses of $m_{1 x}=0.770 \mathrm{~kg}$ and $m_{1 y}=0.768 \mathrm{~kg}$.

The described experiment is repeated with the load mass shown in Fig. 2 attached to the sensor. Including the attached load mass, the amplitude of the sine function is $75.877 \mathrm{~N}$ for $F_{x}$ and $75.736 \mathrm{~N}$ for $F_{y}$. The mass of the attached load

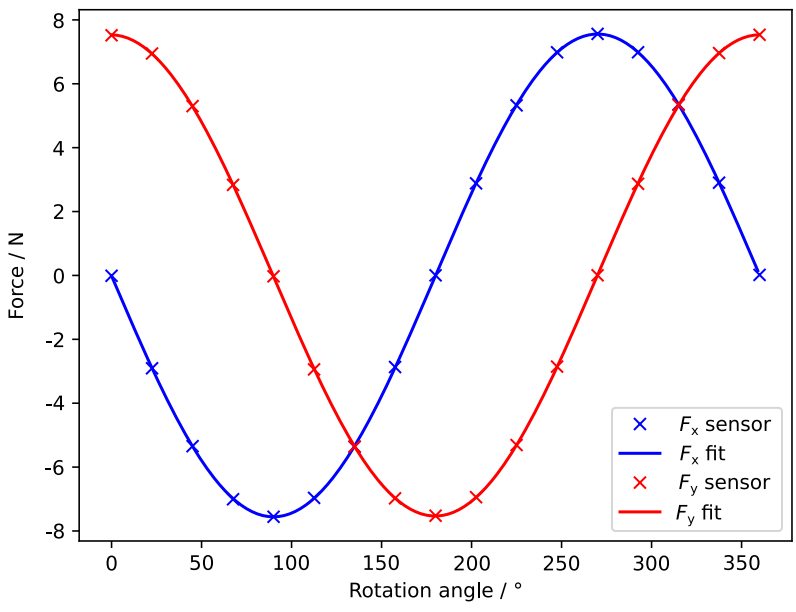

Figure 11. Force readings and sine fitting for $F_{x}$ and $F_{y}$.

mass is calculated according to Eq. (7) to $m_{\mathrm{L} x}=6.962$ and $m_{\mathrm{L} y}=6.951 \mathrm{~kg}$, which differ by $0.028 \%$ and $-0.139 \%$ respectively from the weighted mass $m_{\mathrm{L}}=6.9605 \mathrm{~kg}$.

\subsection{Determination of the spring constant and damping coefficient}

The spring constant is calculated according to the method presented by Schlegel et al. (2012) from the transfer function $H(f)$ as the ratio of the acceleration on the load mass $a_{\mathrm{t}}$ and the acceleration of the shaker surface $a_{b}$ :

$H(f)=\frac{a_{\mathrm{t}}}{a_{b}}$.

The frequency of the maximum of the transfer function represents the resonance frequency $f_{0}$, from which the spring constant can be calculated:

$f_{0}=\frac{1}{2 \pi} \sqrt{\frac{k}{m}}$.

The transfer function $H(f)$ for $F_{z}$, resulting from a periodic chirp excitation with two different load masses $m_{1}=$ $4.236 \mathrm{~kg}$ and $m_{2}=8.798 \mathrm{~kg}$, is shown in Fig. 12. Resonance frequencies are identified at $f_{0,1}=1477.35 \mathrm{~Hz}$ and $f_{0,2}=1092.97 \mathrm{~Hz}$. With the additional internal mass $m_{i}=$ $0.769 \mathrm{~kg}$, the spring constant according to Eq. (9) is calculated to be $k_{1}=4.313 \times 10^{8} \mathrm{Nm}^{-1}$ and $k_{2}=4.512 \times$ $10^{8} \mathrm{~N} \mathrm{~m}^{-1}$.

Figure 13 shows the transfer function $H(f)$ for excitation in $F_{x}$ and $F_{y}$. Because of the horizontal installation of the sensor (Fig. 2), the transfer function is calculated as the ratio of the acceleration of the load mass and the acceleration of the mounting adapter connected to the sensor. The figure shows two resonance peaks for each load mass, which can be explained by the mounting set-up. The connection between the horizontal mounting adapter connected to the sensor and 

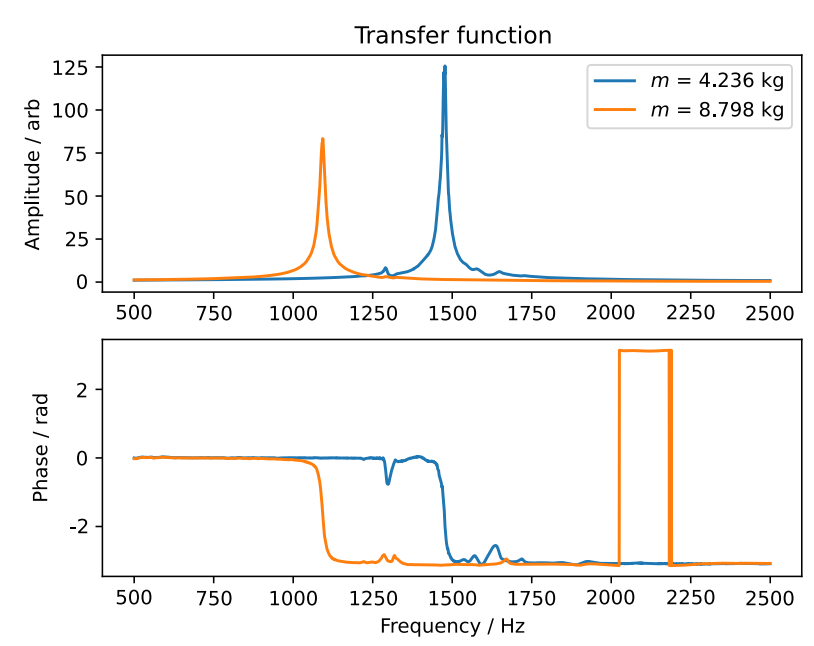

Figure 12. Amplitude and phase of the transfer function $H(f)$ for excitation in $F_{z}$.

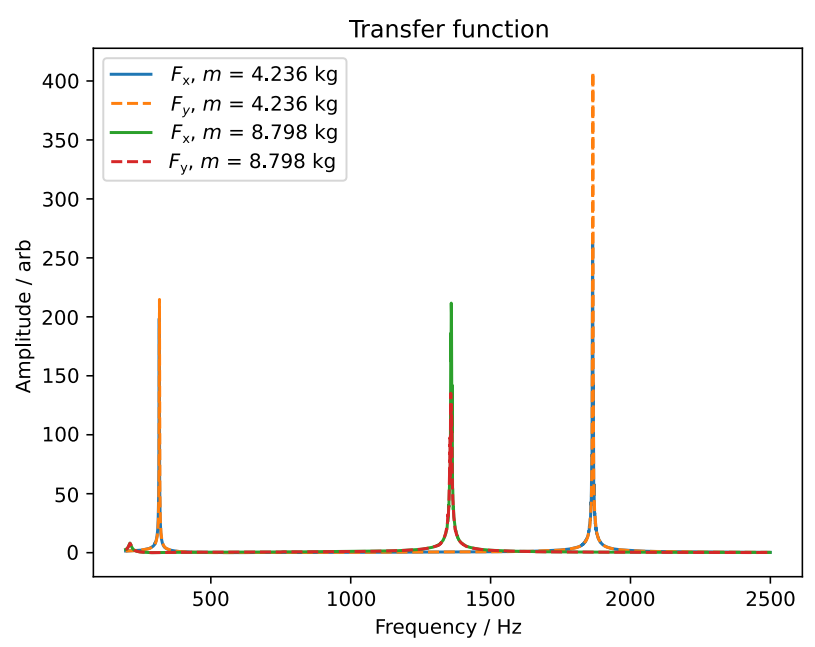

Figure 13. Amplitude of the transfer function $H(f)$ for excitation in $F_{x}$ and $F_{y}$.

the angular adapter introduces a second spring-damper system into the model. It can be seen that the resonance frequencies are identical for excitation in $F_{x}$ and $F_{y}$, while the amplitudes of the resonance peaks differ for the higher resonance frequencies. The identified resonance frequencies and the resulting spring coefficients are listed in Table 2.

In Schlegel et al. (2012), the damping coefficient of the spring-damper model is calculated using the ratio of the resonance frequency and the width of the resonance peak of the transfer function at half the amplitude:

$\frac{f_{0}}{\Delta f_{1 / 2}} \approx \frac{1}{b} \sqrt{k \cdot m}$

For the given transfer function in Fig. 12, the damping coefficients are calculated to be $b_{1}=699.18 \mathrm{~kg} \mathrm{~s}^{-1}$ and $b_{2}=$
$1566.01 \mathrm{~kg} \mathrm{~s}^{-1}$. The damping coefficients for excitation in $x$ and $y$ are listed in Table 2 .

\subsection{Determination of the dynamic sensitivity and phase shift}

To calculate the dynamic sensitivity of the sensor, sinusoidal excitations at different frequencies are used. Measurements are performed at 12 different frequencies from 53.7 to $1020.3 \mathrm{~Hz}$. The sensitivity $S$ is calculated from the ratio of the amplitude of the sensor signal $\hat{s}$, the top mass $m_{\mathrm{t}}$ and the amplitude of the top mass acceleration $\hat{a}_{\mathrm{t}}$ :

$S=\frac{\hat{s}}{\hat{a}_{\mathrm{t}} \cdot m}$.

The acceleration $\hat{a}_{\mathrm{t}}$ is measured at eight different positions on the top mass using the laser vibrometer.

The phase shift between the acceleration of the top mass $a_{\mathrm{t}}$ and the sensor signal $s$ is calculated from the phase of the sine fitting functions of the sensor and the vibrometer signal. The synchronization between the signal sources is performed using the camera trigger signal as described in Sect. 3.2.

Figure 14 shows the mean value of the dynamic sensitivity and the phase shift over the eight acceleration measurements and the resulting standard deviation. The static sensitivity from the sensor calibration data is given as a reference. A rocking mode occurs at a frequency of $375.9 \mathrm{~Hz}$, which leads to higher deviations for the acceleration measurements in the outer areas of the load mass. The rocking motion can also be seen in the higher standard deviation of the phase shift values. Up to the excitation frequency of $1000 \mathrm{~Hz}$, the sensitivity drops to $95.3 \%$ of the static value. The phase shift drops to $-0.25 \mathrm{rad}$ at $800 \mathrm{~Hz}$. For higher frequencies up to $1000 \mathrm{~Hz}$, the phase shift does not change significantly.

\section{Conclusions}

An extended set-up for the dynamic calibration of multicomponent sensors for force and moment measurement has been described. It is based on the periodic acceleration of a sensor and an attached load mass on an electrodynamic shaker. In comparison to earlier works, the design of the load mass and adapting elements was focused on activating single force and moment components where possible. Force components can be activated using a load mass with its centre of gravity in the origin of the sensor coordinate system. For the moment components, a beam cross with a movable load mass was designed.

To identify the movement of the load mass with 6 degrees of freedom, a photogrammetric set-up was installed in the existing laboratory set-up. Two stereo camera systems observe the load mass and the shaker surface. From the displacement of the observed surfaces and the time stamp of the camera images, accelerations can be calculated. With the additional in- 
Table 2. Resonance frequencies, spring constants and damping coefficients for force excitation in $x$ and $y$.

\begin{tabular}{lrrrr|rrrr}
\hline & \multicolumn{4}{c|}{$m_{1}=4.236 \mathrm{~kg}$} & \multicolumn{4}{c}{$m_{2}=8.798 \mathrm{~kg}$} \\
\cline { 2 - 8 } Parameter & $f_{1 x}$ & $f_{1 y}$ & $f_{2 x}$ & $f_{2 y}$ & $f_{1 x}$ & $f_{1 y}$ & $f_{2 x}$ & $f_{2 y}$ \\
\hline Res. frequency $(\mathrm{Hz})$ & 1864.8 & 1865.6 & 315.6 & 316.4 & 1359.4 & 1357.8 & 210.9 & 210.9 \\
Spring constant $\left(10^{8} \mathrm{Nm}^{-1}\right)$ & 6.872 & 6.878 & 0.197 & 0.198 & 6.980 & 6.963 & 0.168 & 0.168 \\
Damping coefficient $\left(\mathrm{kg} \mathrm{s}^{-1}\right)$ & 144.5 & 75.5 & 58.58 & 57.61 & 390.16 & 521.88 & 1003.04 & 1026.59 \\
\hline
\end{tabular}

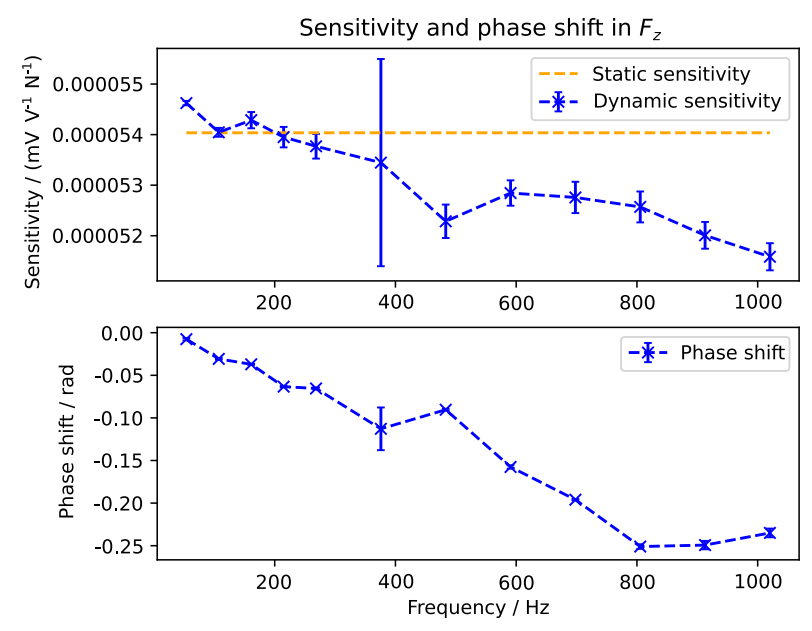

Figure 14. Sensitivity of $F_{z}$ and phase shift between top mass acceleration and sensor signal. Error bars represent the standard deviation for the acceleration at eight different positions on the top mass.

formation of the excitation frequency and reference measurements using a laser interferometer, the displacement of the set-up can be calculated even with camera frame rates lower than the excitation frequency. From an experimental evaluation, a deviation of $0.68 \%$ of the acceleration was achieved for an excitation frequency of $f_{\mathrm{ex}}=106.25 \mathrm{~Hz}$.

The dynamic parameters of the sensor are identified based on a three-dimensional mass-spring-damper system. The internal mass of the sensor was calculated in a static manner from measurements at different rotation angles around the $z$ axis. The resulting internal masses of $x=0.77$ and $y=0.768 \mathrm{~kg}$ differ by $0.3 \%$. The spring constant was calculated from a transfer function as the ratio of the top mass acceleration and the acceleration of the shaker surface. For two different load masses, the resulting spring constant differs by $4.4 \%$ for $F_{z}$. The spring constant for excitation in the $x$ and $y$ directions shows small deviations of less than $0.5 \%$ for the different orientations with the same load mass, while the difference at different load masses for the same orientation rises to up to $15 \%$. It has to be pointed out that the adapting elements for excitation in the $x$ and $y$ directions are still subject to optimization. At frequencies from 350 to
$500 \mathrm{~Hz}$, pitching motions occur which show an influence on the measurements.

The dynamic sensitivity of the sensor was analysed for the $F_{z}$ component. In this set-up, a rocking motion is visible at $375.9 \mathrm{~Hz}$, which increases the standard deviation of the measured accelerations on the top mass. Up to a frequency of $1000 \mathrm{~Hz}$, the dynamic sensitivity drops to $95.3 \%$ of the static value. The phase shift between the sensor signal and the top mass acceleration drops to $-0.25 \mathrm{rad}$ at frequencies of $800 \mathrm{~Hz}$.

As a next step, the analysis performed will be extended to the moment excitation. The previously described beam cross and moment excitation mass are currently being manufactured. Further optimization of the horizontal set-up to reduce pitching motions is suggested to reduce signal crosstalk. Alternatively, a three-dimensional shaker set-up can be used in combination with the adapting element for $F_{z}$ excitation. Finally, the measurement uncertainty for the different set-ups will be analysed.

Data availability. Datasets used in Sects. 3.3 and 5.2 are available at the PTB's public repository https://doi.org/10.7795/720.20181105 (Nitsche et al., 2018).

Author contributions. JN designed and installed the setup, performed measurements and evaluation and wrote the manuscript. RK and RT raised the research problem, supervised the research, and discussed and proofread the paper.

Competing interests. Author Rainer Tutsch is a member of the editorial board of the journal.

Acknowledgements. The authors gratefully acknowledge the funding of this work by the Deutsche Forschungsgemeinschaft (DFG) under grants Tu 135/24 and Ku 3367/1. The authors thank Thomas Bruns and Leonard Klaus of the PTB for assistance with the dynamic moment model, sine fitting and hardware.

Edited by: Ulrich Schmid

Reviewed by: two anonymous referees 


\section{References}

Arun, K. S., Huang, T. S., and Blostein, S. D.: Least-squares fitting of two 3-D point sets, IEEE T. Pattern Anal., PAMI-9, 698-700, https://doi.org/10.1109/TPAMI.1987.4767965, 1987.

Bartoli, C., Beug, M. F., Bruns, T., Elster, C., Esward, T., Klaus, L., Knott, A., Kobusch, M., Saxholm, S., and Schlegel, C.: Traceable dynamic measurement of mechanical quantities: Objectives and first results of this European project, Int. J. Metrol. Qual. Eng., 3, 127-135, https://doi.org/10.1051/ijmqe/2012020, 2012.

Baumgarten, S., Kahmann, H., and Röske, D.: Metrological characterization of a $2 \mathrm{kNm}$ torque standard machine for superposition with axial forces up to $1 \mathrm{MN}$, Metrologia, 53, 1165-1176, https://doi.org/10.1088/0026-1394/53/5/1165, 2016.

Bruns, T., Kumme, R., Kobusch, M., and Peters, M.: From oscillation to impact: The design of a new force calibration device at PTB, Measurement, 32, 85-92, https://doi.org/10.1016/S02632241(01)00048-3, 2002.

Dewetron: DAQP-BRIDGE-B Module Technical reference manual, available at: https://ccc.dewetron.com (last access: 10 October 2018), 2012.

DIN 51309: Werkstoffprüfmaschinen - Kalibrierung von Drehmomentmessgeräten für statische Drehmomente, National standard, DIN, 2005.

Eggert, D. W., Lorusso, A., and Fisher, R. B.: Estimating 3-D rigid body transformations: A comparison of four major algorithms, Mach. Vision Appl., 9, 272-290, https://doi.org/10.1007/s001380050048, 1997.

ISO 376: Metallic materials: Calibration of force-proving instruments used for the verification of uniaxial testing machines, International standard, ISO, Geneva, 2011.

Kim, G.-S.: The development of a six-component force/moment sensor testing machine and evaluation of its uncertainty, Meas. Sci. Technol., 11, 1377-1382, https://doi.org/10.1088/09570233/11/9/318, 2000.

Klaus, L.: Model parameter identification from measurement data for dynamic torque calibration - Measurement results and validation, ACTA IMEKO, 5, 55-63, https://doi.org/10.21014/acta_imeko.v5i3.318, 2016.

Kumme, R.: Untersuchung eines direkten Verfahrens zur dynamischen Kalibrierung von Kraftmessgeräten: Ein Beitrag zur Verringerung der Messunsicherheit, PTB-Bericht PTB-MA-48, Wirtschaftsverl. NW, Verl. für Neue Wiss, Bremerhaven, 1996.

National Instruments: PXI-449x Specifications, available at: http: //www.ni.com/pdf/manuals/372125f.pdf (last access: 10 October 2018), 2012.

Nitsche, J., Baumgarten, S., Petz, M., Röske, D., Kumme, R., and Tutsch, R.: Measurement uncertainty evaluation of a hexapod-structured calibration device for multi-component force and moment sensors, Metrologia, 54, 171-183, https://doi.org/10.1088/1681-7575/aa5b66, 2017a.
Nitsche, J., Röske, D., and Tutsch, R.: Influence of coordinate system alignment on the calibration of multi-component force and moment sensors, in: IMEKO TC3, TC5, TC22 International Conference 2017, $2017 \mathrm{~b}$.

Nitsche, J., Bruneniece, S., Kumme, R., and Tutsch, R.: Design of a calibration setup for the dynamic analysis of multi-component force and moment sensors, in: Proceedings of the XXII IMEKO World Congress, 2018a.

Nitsche, J., Kumme, R., and Tutsch, R.: Dataset for Dynamic characterization of multi-component sensors for force and moment, Physikalisch-Technische Bundesanstalt (PTB), https://doi.org/10.7795/720.20181105, 2018b.

Pan, B., Qian, K., Xie, H., and Asundi, A.: Two-dimensional digital image correlation for in-plane displacement and strain measurement: A review, Meas. Sci. Technol., 20, 062001, https://doi.org/10.1088/0957-0233/20/6/062001, 2009.

Park, Y.-K., Kumme, R., and Kang, D.-I.: Dynamic investigation of a three-component force-moment sensor, Meas. Sci. Technol., 13, 654-659, https://doi.org/10.1088/0957-0233/13/5/302, 2002a.

Park, Y.-K., Kumme, R., and Kang, D.-I.: Dynamic investigation of a binocular six-component force-moment sensor, Meas. Sci. Technol., 13, 1311-1318, https://doi.org/10.1088/09570233/13/8/320, 2002b.

Park, Y.-K., Kumme, R., Röske, D., and Kang, D.-I.: Columntype multi-component force transducers and their evaluation for dynamic measurement, Meas. Sci. Technol., 19, 115205, https://doi.org/10.1088/0957-0233/19/11/115205, 2008.

Röske, D., Peschel, D., and Adolf, K.: The generation and measurement of arbitrarily directed forces and moments: The project of a multicomponent calibration device based on a hexapod structure, in: Proceedings of the 17th International Conference of IMEKO TC3, 339-349, 2001.

Schlegel, C., Kieckenap, G., Glöckner, B., Buß, A., and Kumme, R.: Traceable periodic force calibration, Metrologia, 49, 224-235, https://doi.org/10.1088/0026-1394/49/3/224, 2012.

Schleichert, J., Rahneberg, I., and Fröhlich, T.: Dynamische Kalibrierung eines Mehrkomponentensensors für Kraft und Drehmoment, Tech. Mess., 83, 131-138, https://doi.org/10.1515/teme2015-0090, 2016.

Vlajic, N. and Chijioke, A.: Traceable dynamic calibration of force transducers by primary means, Metrologia, 53, S136-S148, https://doi.org/10.1088/0026-1394/53/4/S136, 2016. 